Original

\title{
Electroestimulación del nervio tibial posterior para el tratamiento de la vejiga hiperactiva. Estudio prospectivo y controlado
}

\author{
Patricia O. Bellette, Paulo C. Rodrigues-Palma, Viviane Hermann, Cássio Riccetto, Miguel Bigozzi, \\ Juan M. Olivares
}

Servicio de Urología de la Facultad de Medicina de la Universidad estatal de Campinas-Unicamp. Brasil.

\section{Resumen}

Objetivo: Evaluar la eficacia y el impacto en la calidad de vida de mujeres con síntomas clínicos de VH, sometidas a estimulación eléctrica percutánea del nervio tibial posterior.

Material y método: Ensayo clínico randomizado, controlado, en 37 mujeres con síntomas clínicos de VH evaluadas en el Servicio de urología femenina del Hospital de Clínicas de UNICAMP. Las pacientes que presentaron los criterios de inclusión, fueron distribuidas, de forma aleatoria, en dos grupos: 21 pacientes en el grupo de tratamiento (electroestimulación percutánea del nervio tibial posterior) y 16 pacientes en el grupo placebo (sólo colocación de los electrodos sin el paso de corriente eléctrica). Las mujeres fueron interrogadas sobre: historia de síntomas, cirugías previas, presencia de síntomas menopáusicos, frecuencia urinaria, urgencia miccional y nocturia, demostrado mediante diario miccional durante tres días. Se respondió el cuestionario de calidad de vida “Overactive Bladder Questionnaire” (OABq), en el momento de la evaluación inicial y final del tratamiento. El tratamiento consistió en 8 sesiones de electroestimulación, dos veces por semana con duración de 30 minutos cada sesión.

Resultados: El trabajo mostró cambios significativos en los síntomas de frecuencia y nocturia, en el grupo sometido a electroestimulación del nervio tibial posterior $(\mathrm{P}=0003=$ ep 0001, respectivamente). La urgencia miccional y la calidad de vida mejoró en ambos grupos significativamente

Conclusión: la electroestimulación del nervio tibial posterior demostró ser un tratamiento fisioterapéutico eficaz en el tratamiento de los síntomas clínicos y mejoría la calidad de vida de las mujeres con VH.

Palabras Clave: Electroestimulación del Nervio Tibial Posterior. Vejiga Hiperactiva. Cuestionario OABq.

\section{Posterior tibial nerve stimulation in the management of overactive bladder: a prospective and controlled study}

\begin{abstract}
The aim of this study was to evaluate the efficacy of the Posterior Tibial Nerve stimulation in women with overactivity bladder symptoms and the impact in the quality of life using the Overactivity Bladder Questionnaire (OABq).

Methods: thirty seven women from female urology outpatient clinic of the Hospital das Clínicas de Campinas (HC/UNICAMP) were enrolled in this prospective, controlled and randomized clinical trial. The patients were randomly placed in one of two groups:

Treatment group: patients were submitted to tibial posterior nerve electrical stimulation $(\mathrm{n}=21)$ and Sham group: patients had electrodes placed without electricity $(n=16)$. After informed consent the patients underwent a physiotherapeutic evaluation, that included voiding diary and the overactivity bladder questionnaire(OABq). The treatment schedule included eight sessions of electrical stimulation in the posterior tibial nerve, twice a week.

Results: there were significant changes in symptoms such as frequency and nocturia $(\mathrm{p}=0,003$ e $p=0,001)$. The urgency and quality of life was improved in both group after treatment.

Conclusion: The posterior tibial nerve electrical stimulation is an effective treatment in overactive bladder.

Keywords: Electrical Stimulation in Posterior Tibial Nerve; Overactive Bladder; OABq.
\end{abstract}

$\mathrm{L}$ a vejiga Hiperactiva (VH) está definida por la International Continence Society (ICS) como un conjunto de síntomas sugestivos de disfunción del tracto urinario inferior. Se trata de una condición clínica en la que la paciente relata la presencia de urgencia miccional, esté o no asociado a incontinencia de urgencia, la mayoría de las veces acompañada por polaquiuria y nocturia ${ }^{1}$. 
La prevalencia estimada de VH varía bastante de acuerdo a diferentes estudios, van de 3\% al 43\% de población ${ }^{1,2}$. En un estudio de prevalencia realizado en Brasil, Teloken ${ }^{3}$ encontró 23,2\% de las mujeres con sintomas irritativos de $\mathrm{VH}$.

$\mathrm{El}$ enfoque de la paciente con vejiga hiperactiva tiene por objetivo, el control de los síntomas clínicos presentados, y proporcionar una mejoría en su calidad de vida a través de la medicación, terapias intravesicales, neuromodulación o fisioterapia.

La estimulación del nervio tibial posterior a través de electrodos de superficie fue propuesta por McGuire $^{4}$ en 1983. En 1987 Stoller et al. ${ }^{5}$ demostró que la estimulación periférica intermitente sobre el nervio tibial posterior (SANS), en simios, podría inhibir la vejiga hiperactiva y la incontinencia de urgencia. Posteriormente, Amarenco ${ }^{6}$ utilizó este tipo de corriente en el tratamiento de la vejiga hiperactiva, a través de electrodos de superficie, y observó en el estudio urodinamico, un aumento de la capacidad vesical, además de retrasar la aparición de contracciones no inhibidas del detrusor.

El objetivo de este estudio prospectivo y controlado fue evaluar el impacto de la estimulación eléctrica percutánea del nervio tibial posterior en la calidad de vida de las mujeres que sufren síntomas clínicos de $\mathrm{VH}$, que fueron sometidas a tratamiento, con respecto al grupo con tratamiento simulado, utilizando como herramienta el cuestionario de “Overactive Bladder Questionaire” (OABq-SF).

\section{MATERIAL Y MÉTODO}

Fueron seleccionadas 37 mujeres con sintomas clínicos de $\mathrm{VH}$, de los consultorios de urología femenina del Hospital de Clínicas de UNICAMP. Las pacientes fueron separadas en dos grupos: 21 pacientes en el grupo de tratamiento y 16 pacientes en el grupo placebo (electrodos colocados pero sin paso de corriente eléctrica).

Los criterios de inclusión en el estudio fueron: edad entre 18 y 85 años, presencia de sintomas de vejiga hiperactiva durante más de seis meses, frecuencia miccional superior a ocho micciones diarias, episodios de nocturia y/o urgencia miccional. Los criterios de exclusión fueron mujeres embarazadas, problemas neurológicos, distopías acentuadas (estadios II o III en la definición de la ICS), infección urinaria e incontinencia urinaria de esfuerzo.

La metodología de trabajo consistió en una evaluación fisioterapéutica y en el tratamiento de elec- troestimulación. Las mujeres fueron interrogadas sobre: la historia de sintomas, de cirugias previas, presencia de sintomas menopáusicos, frecuencia urinaria, urgencia miccional y nocturna, demostrado mediante diario miccional durante tres días. El cuestionario de calidad de vida $\mathrm{OABq}$, se respondió en el momento de la evaluación inicial y final del tratamiento.

El tratamiento consistió en 8 sesiones de electroestimulación percutánea del nervio tibial posterior con un dispositivo Dualpex 961, dos veces por semana con duración de 30 minutos cada uno y el posicionamiento de los electrodos se llevó a cabo según Amarenco ${ }^{6}$.

\section{RESULTADOS}

La edad, la presencia de síntomas menopáusicos, las cirugías previas para incontinencia urinaria y el tiempo de duración de los síntomas, no presentaron diferencias entre los 2 grupos. La edad de las mujeres que participaron en este estudio fue de 47,73 años (+/-10.90). En el momento de la evaluación, $23(62,16 \%)$ estaban en periodo menopáusico, cuatro $(10,81 \%)$ tenían antecedentes de cirugía previa para incontinencia urinaria y ninguna se encontraba en tratamiento farmacológico para la $\mathrm{VH}$. El tiempo promedio de los sintomas de $\mathrm{VH}$ fue 6,86 años $(+/-7,06)$.

En la evaluación inicial la urgencia miccional, estuvo presente en 15 (93,75\%) mujeres del grupo de placebo y en la evaluación final solo en 10 $(62,5 \%)(p=0,025)$. En el grupo de tratamiento, inicialmente $19(90,48 \%)$ mujeres se quejaron de urgencia y en la evaluación final, solo 9 (42.86) mantuvieron las quejas ( $p=0,002$ ) (Tabla 1 ).

En relación a la frecuencia urinaria, el grupo placebo mostró en la evaluación inicial 13,88 micciones por día y al final 10,55. El grupo de tratamiento presentó inicialmente 11,35 y al final 8,29 micciones diarias. Estos resultados, fueron analizados por medio del test de ANOVA y demostró que la disminución de la frecuencia miccional fue estadísticamente signifi-

Tabla 1. Porcentaje de mujeres con urgencia miccional pre y post tratamiento en cada grupo

\begin{tabular}{lcccc}
\hline & $\begin{array}{c}\text { Placebo (\%) } \\
(\mathbf{N}=\mathbf{1 6})\end{array}$ & Valor de $\mathbf{p}^{*}$ & $\begin{array}{c}\text { Tratamiento (\%) } \\
(\mathbf{N = 2 1 )}\end{array}$ & Valor de $\mathbf{p}^{*}$ \\
\hline Inicial & 93,8 & & 90,5 & \\
Final & 62,50 & 0,025 & 42,9 & 0,002 \\
\hline
\end{tabular}

*teste de McNemar 
cativa en el grupo de tratamiento $(\mathrm{p}=0,003)$. El grupo de tratamiento, al final de la intervención tuvo valores más bajos de frecuencia urinaria cuando se compararon con el grupo placebo $(\mathrm{p}=0,009)$ (Fig 1).

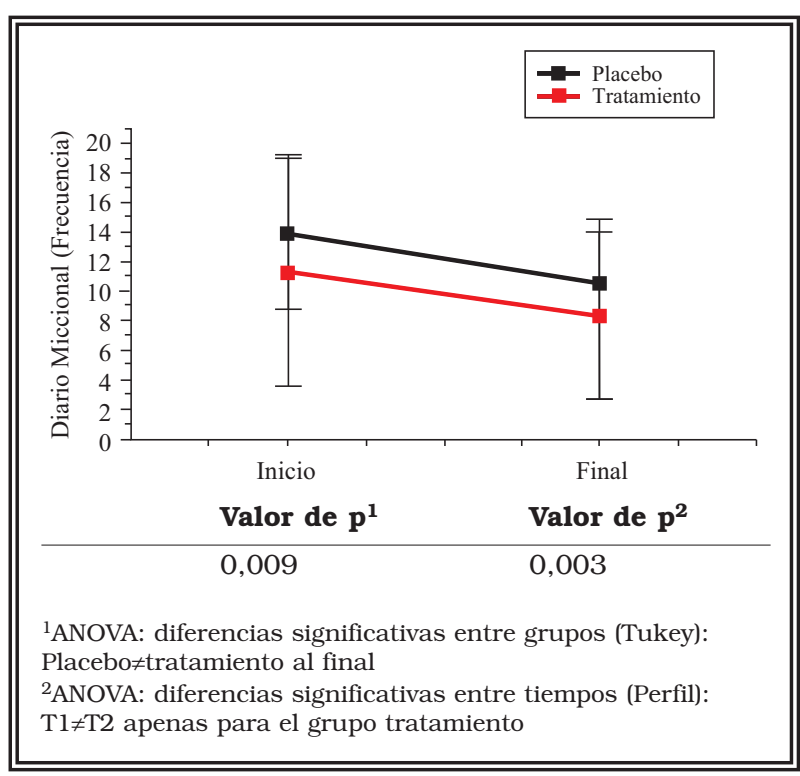

FIGURA 1. Evolución de frecuencia urinaria

La nocturia también presentó disminución a lo largo del tratamiento. En el grupo placebo fue de 2,56 a 2,06 micciones en la noche y el grupo de tratamiento fue 2,38 a 1,14 micciones en la noche. Los resultados de la nocturia, analizados por el test de ANOVA, presentaron diferencias significativas en el grupo de tratamiento $(\mathrm{p}=0,001)$, indicando una tendencia de mejoría en este grupo $(\mathrm{p}=0,054)$ en comparación con el grupo placebo (Fig. 2).

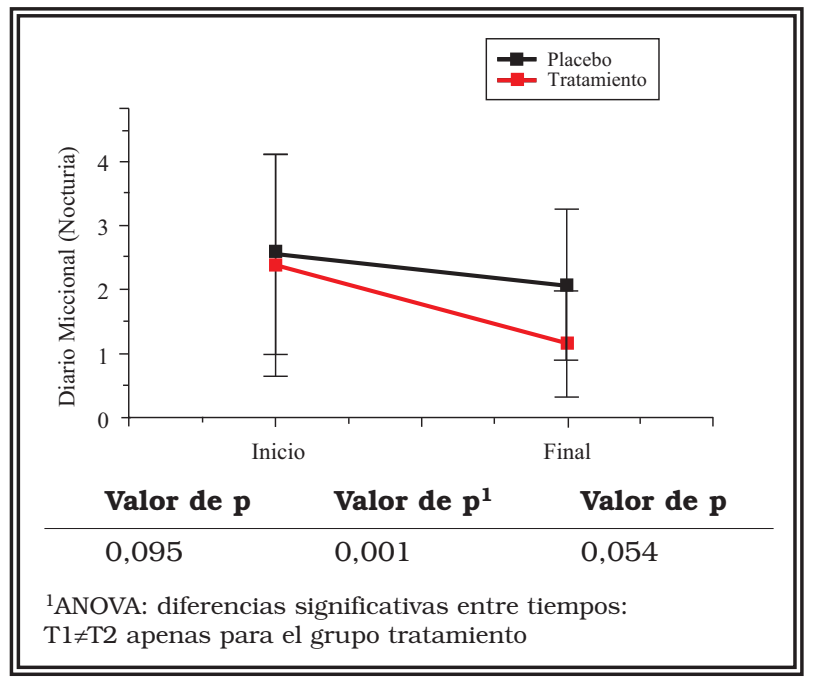

FIGURA 2. Evolución de la nocturia.
En relación al cuestionario $\mathrm{OABq}$, los parámetros evaluados son la severidad y la puntuación total.

En los resultados de severidad, el grupo placebo mostró una puntuación inicial de 67,47 y final de 51,21 , en el grupo de tratamiento 68,55 y 31,72 respectivamente. Los dos grupos analizados mostraron una mejoría estadísticamente significativa $(p<0,001)$, pero el grupo de tratamiento mejoró más que el grupo placebo ( $\mathrm{p}=0,018)$ (Tabla 2 y Fig. 3 ).

En los resultados totales del grupo placebo la puntuación total fue 50,93 a 66,63 y el grupo de tratamiento fue 52,25 a 83,99. Los dos grupos analizados presentaron una mejoría estadísticamente significativa $(\mathrm{p}<0,001)$, pero el grupo de tratamiento mejoró más que el grupo placebo $(\mathrm{p}=0,037)$ (Tabla 3 y Fig. 4).

\section{DISCUSIÓN}

En este ensayo clínico controlado y casi randomizado, se comparó la calidad de vida y los síntomas clínicos de la vejiga hiperactiva, de dos grupos de mujeres. El grupo de tratamiento, sometidas a electroestimulación percutánea del nervio tibial posterior, y el grupo "placebo".

El impacto negativo en la calidad de vida es bien conocido entre las mujeres con vejiga hiperactiva. Para reducir este impacto ellas optan por cambiar sus hábitos de vida, modificar planes de viajes, buscar lugares de fácil acceso a los baños y, a menudo, restricción de líquidos.

El uso de cuestionarios, genéricos o específicos, como instrumentos para evaluar la calidad de vida, se ha intensificado en los últimos años debido al gran interés subjetivo de evaluación clínica, valorando la opinión del paciente sobre su estado de salud. Ellos son importantes para completar una brecha existente entre los métodos de evaluación objetiva, como el diario miccional, el test del pañal y el estudio uro dinámico, que reflejan la gravedad de la pérdida de orina, pero no expresan los cambios en las actividades diarias.

En este estudio se utilizó el Cuestionario de vejiga hiperactiva $(\mathrm{OABq})$, por ser un cuestionario específico, es más sensible para detectar los signos clínicos de vejiga hiperactiva.

La calidad de vida analizada a través de $\mathrm{OABq}$ demostró ser una herramienta eficaz para discriminar el impacto de la vejiga hiperactiva sobre la calidad de vida. Los resultados disminuyeron significa- 
Tabla 2. Variación del score de severidad del cuestionario OABq

\begin{tabular}{|c|c|c|c|c|c|c|c|}
\hline & Placebo & $\pm \mathrm{DP}$ & Tratamiento & $\pm \mathrm{DP}$ & Valor de $\mathbf{p}^{1}$ & Valor de $\mathbf{p}^{2}$ & Valor de $\mathbf{p}^{3}$ \\
\hline OABq inicial & 67,47 & 20,68 & 68,55 & 18,90 & & & \\
\hline OABq final & 51,21 & 32,11 & 31,72 & 18,25 & 0,162 & $<0,001$ & 0,018 \\
\hline Delta $t$ & $-16,26$ & 26,24 & $-36,03$ & 25,33 & & & 0,029 \\
\hline
\end{tabular}

${ }^{2}$ ANOVA: diferencias significativas entre tiempos (Perfil): $\mathrm{T} 1 \neq \mathrm{T} 2$ para ambos grupos.

${ }^{3}$ ANOVA: diferencias significativas entre grupos (Tukey): Placebo $\neq$ tratamiento T2

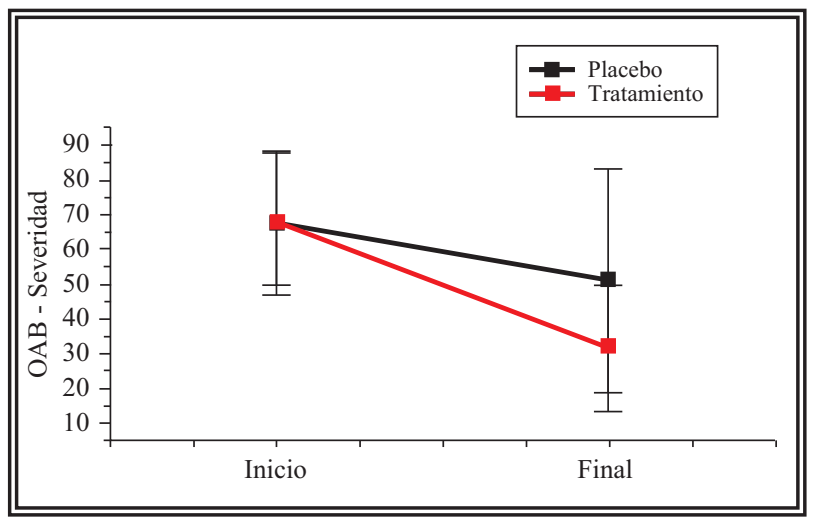

FIGURA 3. Evolución del score de severidad del OABq.

tivamente entre los dos grupos a lo largo del tiempo, pero la mejoría fue más significativa en el grupo de tratamiento.

La electroestimulación del nervio tibial posterior se eligió como método fisioterapéutico, por ser una alternativa interesante para el tratamiento de la vejiga hiperactiva, siendo eficaz y sin efectos colaterales $^{9}$.

Por otra parte, a pesar de que actualmente, el tratamiento farmacológico debe ser la primera opción en el tratamiento de mujeres con síntomas clínicos de vejiga hiperactiva, la adherencia al tratamiento es baja, especialmente por los efectos secundarios que conducen a la suspensión del $60 \%$ de los casos ${ }^{10,11}$.

La electroestimulación del nervio tibial posterior es considerada como una forma de estimulación periférica sacra, más sencilla, mínimamente invasiva, de fácil aplicación, bien tolerado por las pacientes y más accesible económicamente ${ }^{9}$.
Sin embargo, estudios prospectivos y comparativos son escasos en la literatura, en especial mediante herramientas específicas y análisis estadísticos adecuados.

Los resultados que aquí se presentan son una propuesta básica, sobre dudas acerca de las técnicas recomendadas por los fisioterapeutas. Estas dudas son frecuentes, sobre todo en la colocación de los electrodos, tipos de corriente, resultados obtenidos y repercusiones en la calidad de vida de la mujer.

Todas las mujeres se sometieron a ocho sesiones de terapia, todos los cuestionarios fueron completados y ninguna de ella falto a las sesiones más de 3 veces. Las razones por las que faltaron fueron muy variables, no alterando los resultados del estudio. Las evaluaciones se llevaron a cabo por el investigador o la especialista en fisioterapia y el tratamiento lo realizó la misma persona que evalúo, generando un vínculo con el fisioterapeuta.

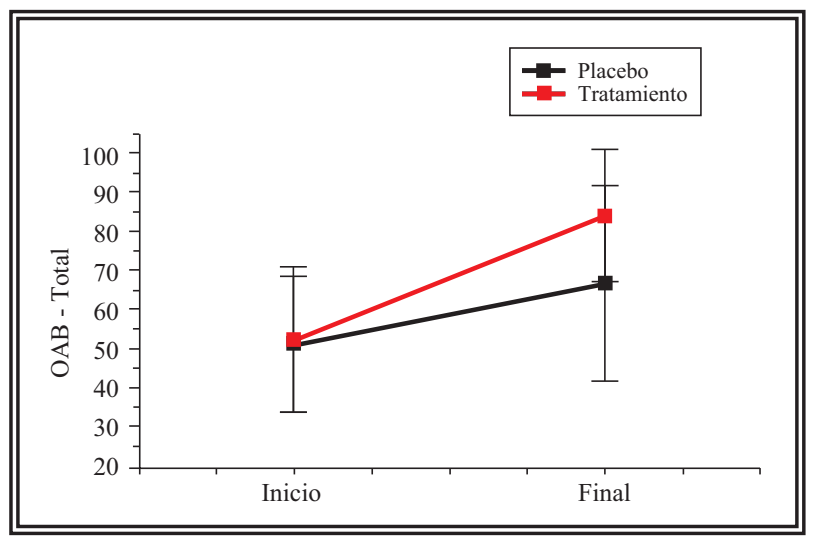

FIGURA 4. Evolución del score total del OABq.

Tabla 3. Variación del score total del cuestionario OABq

\begin{tabular}{lccccccc}
\hline & Placebo & $\mathbf{\pm D P}$ & Tratamiento & \pm DP & Valor de $\mathbf{p}^{\mathbf{1}}$ & ${\text { Valor de } \mathbf{p}^{\mathbf{2}}}^{\mathbf{V a l o r}_{\text {de }} \mathbf{p}^{\mathbf{3}}}$ \\
\hline OABq inicial & 50,93 & 17,42 & 52,25 & 18,62 & & & \\
OABq final & 66,63 & 25,06 & 83,99 & 16,99 & 0,085 & $<0,001$ & 0,037 \\
Delta t & 15,71 & 19,46 & 31,73 & 23,44 & & & 0,023 \\
\hline
\end{tabular}

${ }^{2}$ ANOVA: diferencias significativas entre tiempos (Perfil): $\mathrm{T} 1 \neq \mathrm{T} 2$ para ambos grupos.

${ }^{3}$ ANOVA: diferencias significativas entre grupos (Tukey): Placebo $\neq$ tratamiento T2 
Podemos observar disminución significativa en el grupo de tratamiento en las variables de frecuencia y nocturia, evidenciando superioridad de este grupo en relación con el grupo placebo.

Se encontró en este estudio, normalización de la frecuencia urinaria en las mujeres en el grupo de tratamiento de 11 a 8 micciones diarias. Estos resultados, fueron analizados por el test ANOVA que demostró una disminución de la frecuencia miccional estadísticamente significativa en el grupo de tratamiento ( $\mathrm{p}=0,003$ ). El grupo de tratamiento, al final de la evaluación tuvo valores menores en la frecuencia urinaria, en comparación con el grupo placebo $(\mathrm{p}=0,009)$.

En los estudios de Van der Pal et $\mathrm{al}^{12}$ también observó disminución en el número de micciones diarias, además de la disminución de los apósitos usados en los episodios de pérdida de orina. Fall $^{13}$ (1985) utilizó este recurso en el tratamiento de la cistitis intersticial, obteniendo buenos resultados en los síntomas y un mayor intervalo entre micciones.

Cuando se evaluó la variable nocturia, observamos que las mujeres en el grupo de tratamiento tuvieron disminución de dos a un episodio por noche, siendo considerado un valor normal $(\mathrm{p}=$ 0,001).

La literatura muestra que la nocturia es uno de los sintomas que causa mayor impacto en la calidad de vida. Coyne $^{7}$ (2003) utilizó el OABq para evaluar los sintomas de nocturia, en la calidad de vida y observó una interferencia con el sueño y en la interacción social.

La urgencia, el principal síntoma de la vejiga hiperactiva, estuvo presente en aproximadamente el 90\% de las mujeres incluidas en este estudio. Las mujeres que no presentaron este síntoma fueron incluidas por presentar un aumento en la frecuencia urinaria y nocturia.

Los resultados demostraron que la estimulación del nervio tibial fue eficaz en ambos grupos, cuando la variable evaluada fue la urgencia miccional. En el grupo de tratamiento, la reducción fue de $90 \%$ a $43 \%$ (p-valor $=0,002$ ) y el grupo placebo fue de $93 \%$ a $62,5 \%$ (p-valor $=0,025)$.

McGuire $^{4}$ (1983) en su estudio de 22 mujeres con síntomas clínicos de vejiga hiperactiva, encontró 12 mujeres continentes, que corresponde a 55\% y $7(32 \%)$ con mejoría, después del tratamiento con estimulación eléctrica del nervio tibial posterior.
En este estudio, hemos observado alrededor de $50 \%$ de mejoría en el grupo de tratamiento, coincidiendo con la literatura, en la cual son presentados estudios con tasas de mejoría de alrededor de un $50 \%$ a un $70 \%$ de los pacientes ${ }^{8,9}$.

Podemos justificar esta mejoría a través del estudio de Amarenco ${ }^{6}$ (2003) que observó durante la evaluación urodinámica, un aumento de la capacidad vesical, además de un retardo en la aparición de contracciones no inhibidas del detrusor. Estos dos factores pueden actuar disminuyendo la urgencia miccional presentada por la mujer.

IRWIN et $a^{13}$. (2005) observó, en su trabajo, que el 32\% de las personas entrevistadas con síntomas de vejiga hiperactiva, presentaban sintomas de depresión y el 28\% síntomas de estrés. Además, el $76 \%$ informó que los síntomas interferían negativamente en su vida diaria.

El cuestionario de calidad de vida OABq, demostró ser un cuestionario sensible para detectar los signos clínicos de vejiga hiperactiva y la influencia en la calidad de vida de las mujeres.

La mejoría en la calidad de vida, después de la estimulación percutánea del nervio tibial posterior, fue significativa tanto en la puntuación de severidad y en la puntuación total del OABq en ambos grupos, coincidiendo con la literatura.

Tanto el grupo de tratamiento, como el grupo placebo, presentó mejoría en los scores de calidad de vida, scores de severidad y total $(\mathrm{p}<0,05)$, pero el grupo de tratamiento presentó superioridad en relación con el grupo placebo en ambos scores: severidad (0018) y total ( $\mathrm{p}=0037)$.

En algunos estudios, evaluaron la calidad de vida y los sintomas clínicos de la vejiga hiperactiva, después del tratamiento con estimulación del nervio tibial posterior, con otros cuestionarios, como el SF36 y IQOL9, 12. En estos estudios, la mejoría de la calidad de vida fue el objetivo primordial para considerar el éxito del tratamiento.

En este estudio, hemos encontrado en el grupo placebo, aproximadamente un 30\% de mejoría en los sintomas de las mujeres, coincidiendo con los resultados encontrados en estudios farmacológicos, que muestran una mejoría aproximadamente del $30 \%$, con el efecto placebo.

\section{CONCLUSIÓN}

La electroestimulación del nervio tibial posterior mostró ser un tratamiento eficiente, seguro, no invasivo, que mejora significativamente los síntomas de VH y la calidad de vida de las pacientes. 


\section{REFERENCIAS}

1. Abrams P. Padronização da terminologia da função do trato urinário inferior. Neurourol Urodyn. 2002;21(2):167-178.

2. Hampel C, Wienhold D, Dahms SE, Thuroff JW. Heterogeneity in epidemiological investigations of bladder control problems: a problem of definition. BJU Int. 1999 Mar;83 Suppl 2:10-5.

3. Teloken C, Caraver F, Weber FA, Teloken PE, Moraes IF, Sogari PR, Graziottin TM. Overactive bladder: prevalence and implications in Brazil. European Urology. 2006., 49(6):1087-1092.

4. McGuire E, Shi-Chun Z, Horwinsk ER, Lytton B. Treatment of motor and sensory detrusor instability by electrical stimulation. J Urology.1983, 129(1):78-79.

5. Stoller M. Afferent nerve stimulation for pelvic floor dysfunction. European Urology, 37(2):33, 2000.

6. Amarenco G, Ismael S, Even-Schneider, Raibaut P, DemailleWlodyka S, Parrate B, Kerdraon J. Urodynamic effect of acute transcutaneous posterior tibial nerve stimulation in overactive bladder. J Urology2003;169(6):2210-2215.

7. Coyne K, Revicki D, Hunt T, Corey R, Stewart W, Bentkoevr J, Kurth H, Abrams P. Psychometric validation of an overactive bladder symptom and health- related quality of life questionnaire: the OAB-Q Qual Life Res. 2002;11(6):563-574

8. Govier F, Litwiller S, Nitti V, Kreder K, Rosenblatt P. Percutaneous afferent neuromodulation for the refractory iveractive bladder: results of a multicenter study. J Urol. 2001;165 (4):1193-1198.
9. Van Balken MR, Vergunst H, Bemelmans BLH. Prognostic factors for successful percutaneous tibial nerve stimulation Eur Urol. 2006;49(2):360-365

10. Abrams P, Kelleher CJ,Kerr LA, Rogers RG. Overactive bladder significantly affects quality of life. Am J Manag Care. 2000 Jul; 6(11 Suppl):S580-590

11. Chapple C. The contemporary pharmacological management of overactive bladder. Journal of obstetrics and gynecology, 19-28, 2006.

12. Van der Pal F, Van Balken MR, Heesakkers JPFA, Debruyne FMJ, Kiemeney LALM, Bemelmans BLH. Correlations between quality of life and voiding variables in patients treated with percutaneous tibial nerve stimulation. BJU Int. 2006 Jan;97(1): 113-116.

13. Irwin D, Milson I, Kopp Z, Abrams P, Cardozo L. Impact of overactive bladder symptoms on employment, social interactions and emotional well-being in six european countries. BJU Int. 2006;97(1):96-100.

Correspondencia autor: Dr. Paulo Palma

Servicio de Urología de la Facultad de Medicina de la

Universidad estatal de Campinas-Unicamp.

Rua Jose Pugliasi Filho 265

Campinas, São Paulo, Brasil 13085-415

E-mail autor: ppalma@uol.com.br

Información artículo: Original - Urología femenina - Incontinencia

Trabajo recibido: febrero 2008

Trabajo aceptado: septiembre 2008 